\title{
Moving average filters and periodic integration
}

\author{
Philip Hans Franses * , Richard Paap \\ Econometric Institute and Tinbergen Institute, Erasmus University P.O. Box 1738, NL-3000 DR Rotterdam, Netherlands
}

\section{Introduction}

Several quarterly observed macroeconomic time series may contain a stochastic trend which effects the seasonal fluctuations. An example is the unemployment rate which displays seasonality in business cycle expansion periods because of seasonal labor supply, and which shows much less seasonal fluctuations in the contraction periods because the dismissal of employees may be free of seasonal effects. Hence, the seasonal pattern of economic time series can change over time, and these changes may be caused by the stochastic trend. A class of univariate time series models that can describe such time series contains the periodic autoregressions with unit roots [PIAR], see, e.g., $[1,2]$.

The basic assumption underlying seasonal adjustment methods is that, one way or another, seasonality, trend and cycles can be separated. However, when it is found that a PIAR can give an adequate description of a time series, the crucial requirement is violated. Hence, seasonal correction filters may either not remove all seasonal fluctuations or effect trend and cyclical patterns. In this paper, we focus on the effect of one particular filter, i.e. the linear moving average filter $\left(1+B+B^{2}+B^{3}\right)$ on testing for common stochastic trends across periodically integrated time series, where $B$ is the familiar backward shift operator.

The outline of this paper is as follows. In Section 2, we discuss a few concepts related to PIAR processes. In Section 3, we discuss the linear moving average filter in relation to a PIAR. In Section 4, we present the results of some Monte Carlo exercises. The main conclusion is that the probatility of finding true common trends across PIARs is dramatically reduced when moving average filters are used. In Section 5, we conclude with some remarks.

\footnotetext{
* Corresponding author 


\section{Periodic integration}

Consider the time series $y_{t}, t=1, \ldots, n$, which is quarterly observed during $N$ years, i.e., $n=4 N$. A periodically integrated autoregressive process of order 1 [PIAR(1)] is

$$
y_{t}=\phi_{s} y_{t-1}+\varepsilon_{t}
$$

with $\phi_{1} \phi_{2} \phi_{3} \phi_{4}=1$, where $\phi_{s}$ is a parameter which value varies with the season, and where $\varepsilon_{t}$ is a standard white noise process, see [1] and [2]. The restriction $\phi_{1} \phi_{2} \phi_{3} \phi_{4}=1$ is given by the solution of the characteristic equation corresponding to a vector representation of (1),

$$
A_{0} Y_{T}=A_{1} Y_{T-1}+\varepsilon_{T}
$$

where $A_{0}$ and $A_{1}$ are

$$
A_{0}=\left[\begin{array}{cccc}
1 & 0 & 0 & 0 \\
-\phi_{2} & 1 & 0 & 0 \\
0 & -\phi_{3} & 1 & 0 \\
0 & 0 & -\phi_{4} & 1
\end{array}\right], \quad A_{1}=\left[\begin{array}{cccc}
0 & 0 & 0 & \phi_{1} \\
0 & 0 & 0 & 0 \\
0 & 0 & 0 & 0 \\
0 & 0 & 0 & 0
\end{array}\right]
$$

and $Y_{T}$ is the $(4 \times 1)$ vector $\left(Y_{1 T}, Y_{2 T}, Y_{3 T}, Y_{4 T}\right)^{\prime}$, where $Y_{s T}$ is the observation in season $s$ in year $T, T=1, \ldots, N$. The characteristic equation for (2) is

$$
\left|A_{0}-A_{1} z\right|=0
$$

which is equal to $1-\phi_{1} \phi_{2} \phi_{3} \phi_{4} z=0$. Note that $\phi_{1} \phi_{2} \phi_{3} \phi_{4}=1$ implies that there is one unit root in the system $Y_{T}$, or equivalently, that there are three cointegration relations between its elements $Y_{s T}$. In [2] a test is proposed for the hypothesis that $z=1$ is a solution to (3).

For higher order periodic autoregressive processes one can consider similar characteristic equations as (3) to test for a unit root in $Y_{T}$. Sometimes it may however be more convenient to analyze such time series in a differenced form. For example, under the assumption that the solutions to (3) are not complex-valued, a PAR(2) process can be written as

$$
y_{t}-\phi_{s} y_{t-1}=\beta_{s}\left(y_{t-1}-\phi_{s-1} y_{t-2}\right)+\varepsilon_{t}
$$

where $\phi_{0}=\phi_{4}$. Imposing the restriction $\phi_{1} \phi_{2} \phi_{3} \phi_{4}=1$ in (4) implies that there is a unit root in the corresponding vector model. Further, model (4) can be easily used to check the hypothesis $\phi_{s}=\phi=1$, see [3]. If so, one should apply the $(1-B)$ filter to make a time series (periodically) stationary.

From (4) is can be observed that the differencing filter for a PIAR time series varies with the season. This indicates that seasons and trends are not independent. Hence, if one wants to investigate whether two or more periodically integrated time series have a common stochastic trend, one should take account of this dependency. In [4] a method is proposed to test for common trends which contains two steps. The first is the estimation of the most nonstationary linear combination between the $Y_{s T}$ elements for each of the $y_{t}$ series using the method in [5]. The second step is to use the regular cointegration methods to these combinations. Simulation and empirical evidence suggests that this method can yield useful results. A possible drawback of this method is that the second round of cointegration analysis uses only $n / 4$ observations. 


\section{Moving average filters}

In the practical occasions where PIAR models are found to be appropriate, the estimated values of $\phi_{s}$ are usually close to unity, as can be expected from the expressions in (1) and (4). The application of HEGY method in [6] to a process that is generated by a PIAR process is therefore likely to suggest that the differencing filter $(1-B)$ should be applied, although the presence of seasonal unit roots, i.e. that the $\left(1-B^{4}\right)$ filter should be used, can also often not be rejected, see [7]. The latter can be clarified by writing, e.g., (1) as

$$
y_{t}=y_{t-4}+\varepsilon_{t}+\phi_{s} \varepsilon_{t-1}+\phi_{s} \phi_{s-1} \varepsilon_{t-2}+\phi_{s} \phi_{s-1} \phi_{s-2} \varepsilon_{t-3},
$$

see also [8].

When the HEGY method is applied to two quarterly observed time series, say $y_{t}$ and $x_{t}$, and it is found that they both have a nonseasonal unit root, as well as three seasonal unit roots, a usual next step is to check whether the series have a nonseasonal unit root in common. For that purpose, $y_{t}$ and $x_{t}$ are transformed using the $\left(1+B+B^{2}+B^{3}\right)$ filter, see [9] [EGHL]. This is because the polynomial $1-B^{4}$ can be decomposed as $(1-B)\left(1+B+B^{2}+B^{3}\right)$. The new time series, say $y_{1 t}$ and $x_{1 t}$, are then compared in a cointegration exercise. In EGHL it is shown that the standard critical values of [10] apply.

As already indicated in the previous section, the $\left(1+B+B^{2}+B^{3}\right)$ filter does not correspond to the appropriate differencing filter for a PIAR series. This is because it deals with each of the seasons in a similar way, while the main property of a PIAR process is that the seasons display distinct behavior. This can easily be recognized from (5), where it can be seen that a $\left(1+B+B^{2}+B^{3}\right)$ transformed PIAR(1) model results in an $\operatorname{ARIMA}(0,1,3)$ process with periodically varying MA structures. The results in, e.g., [11] suggest that tests for unit roots can be effected by the presence of neglected MA components. A further drawback of applying $\left(1+B+B^{2}+B^{3}\right)$ filter to a PIAR process is that it assumes too many unit roots for the $y_{t}$ series. From a framework as (2), it can readily be derived that a $\left(1+B+B^{2}+B^{3}\right)$ filter assumes that there is one cointegrating relation between the $Y_{s T}$ variables, see also [7]. Hence, in some sense the $\left(1+B+B^{2}+B^{3}\right)$ transformed PIAR series is not an invertible time series. In summary, all this suggests that the standard EGHL type of analysis applied to PIAR processes may yield inappropriate results.

\section{Some simulation results}

To verify the conjecture that the moving average filter has an impact on tests for common trends across PIAR time series, we conduct several Monte Carlo exercises based on 5000 replications using Gauss 386 VMI version 3.1.1. The data generating processes (DGP) are
(i) $y_{t}=\alpha_{s} y_{t-1}+\varepsilon_{t}, \quad x_{t}=\gamma_{s} x_{t-1}+\nu_{t}$,
(ii) $y_{t}=\alpha_{s} y_{t-1}+\varepsilon_{t}, \quad x_{t}=y_{t}+\eta_{t}$,
(iii) $y_{t}=\alpha_{s} y_{t-1}+\varepsilon_{t}, \quad x_{t}=y_{t}+\eta_{t} /(1-0.5 B)$,
(iv) $y_{t}=\alpha_{s} y_{t-1}+\varepsilon_{t}, \quad x_{t}=y_{t}+\eta_{t} /(1-0.8 B)$,
(v) $y_{t}=\alpha_{s} y_{t-1}+\varepsilon_{t}, \quad x_{t}=y_{t}+\eta_{t} /(1-0.9 B)$ 
Table 1

Rejection frequencies of the hypothesis of no cointegration. Based on 5000 replications of sample size 100. Critical values are taken from [10].

\begin{tabular}{|c|c|c|c|c|c|}
\hline $\begin{array}{l}\text { Data generating } \\
\text { process }\end{array}$ & $\begin{array}{l}\text { Nominal } \\
\text { size }\end{array}$ & $\begin{array}{l}\text { EGHL } \\
\text { CRDW }\end{array}$ & $\mathrm{CRDF}^{(1)}$ & $\begin{array}{l}\text { Franses 2-step } \\
\text { CRDW }\end{array}$ & $\mathrm{CRDF}^{(2)}$ \\
\hline \multirow[t]{3}{*}{ (i) } & $5 \%$ & 0.0 & 4.2 & 5.7 & 5.2 \\
\hline & $10 \%$ & 0.0 & 8.7 & 11.8 & 11.3 \\
\hline & $20 \%$ & 0.0 & 15.7 & 20.4 & 21.4 \\
\hline \multirow[t]{3}{*}{ (ii) } & $5 \%$ & 95.4 & 35.7 & 97.4 & 95.2 \\
\hline & $10 \%$ & 99.4 & 48.2 & 99.3 & 98.5 \\
\hline & $20 \%$ & 100,0 & 60.8 & 99.8 & 99.7 \\
\hline \multirow[t]{3}{*}{ (iii) } & $5 \%$ & 4.7 & 32.6 & 95.6 & 91.5 \\
\hline & $10 \%$ & 18.8 & 44.3 & 98.7 & 96.8 \\
\hline & $20 \%$ & 52.7 & 56.8 & 99.7 & 99.3 \\
\hline \multirow[t]{3}{*}{ (iv) } & $5 \%$ & 0.0 & 23.6 & 71.2 & 59.5 \\
\hline & $10 \%$ & 0.1 & 35.5 & 84.6 & 74.9 \\
\hline & $20 \%$ & 1.2 & 47.8 & 93.8 & 88.3 \\
\hline \multirow[t]{3}{*}{ (v) } & $5 \%$ & 0.0 & 15.9 & 38.4 & 29.1 \\
\hline & $10 \%$ & 0.0 & 26.2 & 55.2 & 43.5 \\
\hline & $20 \%$ & 0.1 & 37.7 & 72.5 & 61.9 \\
\hline
\end{tabular}

(1) The lag length in the augmented Dicky-Fuller regression is determined by LM tests for residual autocorrelation.

(2) The lag length here is set equal to zero.

where $\varepsilon_{t}, \nu_{t}$ and $\eta_{t}$ are standard white noise processes, and where $\alpha_{s}, \gamma_{s}$ is set equal to 1.25 , $0.8,2$ and 0.5 for the four seasons. For each of these DGPs we calculate the Durbin-Watson (CRDW) and Dickey-Fuller (CRDF) statistics for the residuals of the regression of $\left(1+B+B^{2}\right.$ $\left.+B^{3}\right) y_{t}$ on $\left(1+B+B^{2}+B^{3}\right) x_{t}$, reflecting the EGHL method, and the same statistics for the residuals of the regression of the two most nonstationary linear combinations of the $Y_{T}$ and $X_{T}$ elements, reflecting the Franses two-step method. The results for DGP (i) can be interpreted as the empirical size of the tests, while the results for the other DGPs correspond to the empirical 'power' of the methods.

The results in this table seem very easy to interpret. The EGHL method yields an incorrect size of the CRDW statistic, while the size of the CRDF test is still reasonable. The size of both test statistics in Franses' 2-step method seems adequate. The (not size-corrected) 'power' of the CRDW test in the EGHL case is only high for DGP (ii) but converges to zero when autocorrelated residuals are allowed. The 'power' of the CRDF test in the EGHL case is low, though it may sometimes adequately suggest the presence of common trends. On the other hand, the values of the CRDW test are likely to be extremely low. The 'power' of the Franses 2 -step method is quite reasonable, even in the case where the errors are strongly autocorrelated.

\section{Concluding remarks}

An application of the moving average filter to periodically integrated autoregressive time series in order to check for common trends across such series cannot be recommended. The 
model selection strategy proposed in [3] may be useful in deciding which filters are the most useful for such series. Whether the simulation results in this paper extend to officially seasonally adjusted time series using, e.g., the Census-X11 method, is a topic for future research.

Notes. This paper was initiated while the first author visited the Institute of Statistics and Econometrics at the Humboldt University Berlin, April 1993. Thanks are due to their generous hospitality and to the Royal Netherlands Academy of Arts and Sciences for its financial support.

\section{References}

[1] D.R. Osborn, Seasonality and habit persistence in a life-cycle model of consumption, J. Appl. Econom. 3 (1988) 255-266.

[2] H.P. Boswijk and P.H. Franses, Testing for periodic integration, Econom. Lett. 48 (1995) 235-240.

[3] P.H. Franses, and R. Paap Model selection in periodic autoregressions, Oxford Bull. Econom. Statist. 56 (1994) 421-439.

[4] P.H. Franses, Testing for common trends across periodically integrated time series, Econometric Institute Report 9320, Erasmus University, 1993.

[5] G.E.P. Box and G.C. Tiao, A canonical analysis of multiple time series, Biometrika 64 (1977) 355-365.

[6] S. Hylleberg, R.F. Engle, C.W.J. Granger and B.S. Yoo, Seasonal integration and cointegration, J. Econom. 44 (1990) 215-238.

[7] P.H. Franses, A multivariate approach to modeling univariate seasonal time series, J. Econom. 63 (1994) 133-151.

[8] D.R. Osborn, Comment on Engle et al., J. Econom. 55 (1993) 300-303.

[9] R.F. Engle, C.W.J. Granger, S. Hylleberg, and H.S. Lee, Seasonal cointegration: The Japanese consumption function, J. Econom. 55 (1993) 275-298.

[10] R.F. Engle and C.W.J. Granger, Cointegration and error correction: Representation, estimation and testing, Econom. 55 (1987) 251-265.

[11] G.W. Schwert, Effects of model specification on tests for unit roots in macroeconomic data, J. Monetary Econom. 20 (1987) 73-103. 